\title{
Pós-graduação brasileira no tratamento de águas residuárias por wetlands construídas
}

Brazilian post-graduation in wastewater treatment by constructed wetlands

Cursos de posgrado brasileño en tratamiento de águas residuales por humedales artificiales

Édio Damásio da Silva Júnior Professor doutor, Instituto Federal Goiano - Rio Verde, Brasil edio.damasio@ifgoiano.edu.br

Marco Antonio Almeida de Souza Professor doutor, Universidade de Brasília, Brasil marcantoniosouza@gmail.com

Márcia Franco de Assis Mestranda, Instituto Federal Goiano - Rio Verde, Brasil marciafrancoassis@gmail.com 
RESUMO

A reduzida divulgação das pesquisas realizadas na pós-graduação (mestrado e doutorado) brasileira versando sobre o uso de wetlands construídas no tratamento de águas residuárias pode ser aventada como uma das causas da limitada utilização dessa tecnologia fora do ambiente acadêmico. O presente estudo objetivou sintetizar as informações das pesquisas em nível de pós-graduação no Brasil sobre o uso de wetlands construídas no tratamento de águas residuárias. Foram levantados dados de 253 dissertações e teses (em 30 anos) em todo o território brasileiro. Algumas conclusões puderam ser encontradas, tal como a constatação de que as instituições acadêmicas da região sul-sudeste foram responsáveis pela maior parte da produção sobre o tema no país. As espécies vegetais do gênero Typha spp e a brita compuseram a vegetação e meio suporte mais utilizados nos trabalhos, respectivamente. As tipologias de águas poluídas mais adotadas pelos pesquisadas foram esgoto proveniente de campus universitário e esgoto sanitário urbano. As terminologias mais utilizadas para indicar esse processo de tratamento de águas residuárias foram respectivamente "wetlands construídas" e "alagados construídos". Estes sistemas de tratamento operaram predominantemente em nível secundário e terciário, avaliando principalmente a remoção de matéria orgânica e nutrientes das águas estudadas.

PALAVRAS-CHAVE: Alagados construídos. Leitos cultivados. Zona de raízes.

\begin{abstract}
The reduced dissemination of researches carried out in brazilian post-graduation studies (master and doctorate) dealing with the use of constructed wetlands in wastewater treatment can be suggested as one of the causes of the limited use of this technology outside the academic studies. The present paper aimed to synthesize information from research at the post-graduation level in Brazil on the use of constructed wetlands in wastewater treatment. Data from 253 dissertations and thesis (over 30 years) were collected throughout the brazilian territory. Thus, some conclusions could be found, such as the observation that academic institutions in the south-southeast region were responsible for most of the production on that subject in the country. The plant species of the genus Typha spp and the gravel composed the vegetation and support media most used in those studies, respectively. The types of polluted water most adopted by those researchers were sewage from the university campus and urban sewage. The terminologies most used to indicate this wastewater treatment process were "wetlands construídas" and "alagados construidos" respectively. These treatment systems operated predominantly at the secondary and tertiary levels, evaluating mainly the removal of organic matter and nutrients from the studied waters.
\end{abstract}

KEYWORDS: Constructed wetlands. Vegetated beds. Zoots zone.

\title{
RESUMEN
}

La difusión reducida de la investigación llevada a cabo en estudios de posgrado brasileños (maestría y doctorado) sobre el uso de humedales construidos en el tratamiento de aguas residuales puede ser sugerida como una de las causas del uso limitado de esta tecnología fuera del entorno académico. El presente estudio tuvo como objetivo sintetizar información de la investigación a nivel de posgrado en Brasil sobre el uso de humedales construidos en el tratamiento de aguas residuales. Se recopilaron datos de 253 disertaciones y tesis (en 30 años) en todo el territorio brasileño. Se pueden encontrar algunas conclusiones, como la observación de que las instituciones académicas en la región sur-sureste fueron responsables de la mayor parte de la producción sobre el tema en el país. Las especies de plantas del género Typha spp y la grava componían la vegetación y el medio de soporte más utilizado en las obras, respectivamente. Los tipos de agua contaminada más adoptados por los encuestados fueron las aguas residuales del campus universitario y las aguas residuales urbanas. Las terminologías más utilizadas para indicar este proceso de tratamiento de aguas residuales fueron "wetlands construídas" y "alagados construídos" respectivamente. Estos sistemas de tratamiento operaron predominantemente en los niveles secundario y terciario, evaluando principalmente la eliminación de materia orgánica y nutrientes de las aguas estudiadas.

PALABRAS CLAVE: Humedales artificiales. Camas cultivadas. Zonas de raízes. 


\section{INTRODUÇÃO}

O uso de wetlands construídas como tecnologia de tratamento para melhorar a qualidade de águas residuárias no Brasil teve origem nos anos 1980. Água para abastecimento humano (proveniente de corpos hídricos superficiais), esgoto sanitário urbano e efluentes líquidos industriais foram os primeiros tipos de águas usadas nas pesquisas. Nesses estudos, os pesquisadores utilizaram a "depuração hídrica com solos" (terminologia adotada na época), constituída por uma camada filtrante de solo sobreposta a camadas de rochas, para tratar a água através da interação solo-planta-microrganismos. Canais com macrófitas aquáticas flutuantes também foram amplamente utilizados (SALATI JÚNIOR et al., 1999).

Ao longo de décadas, o uso de wetlands construídas no Brasil se intensificou, principalmente no ambiente acadêmico, tanto em quantidade como em variedade de configurações de unidades de tratamento. Foram realizados estudos aplicando diferentes tipos de efluentes, meios suportes, espécies de plantas, tipos de fluxo hidráulico e outras configurações, relacionando-os à eficiência do sistema de remoção de poluentes da água. Esses estudos proporcionaram a evolução da tecnologia no Brasil.

Por outro lado, apesar do avanço dos estudos sobre o tema no país, seu uso em larga escala ainda é limitado, restringindo-se às pesquisas universitárias na grande maioria. Segundo o último relatório da Pesquisa Nacional de Saneamento Básico (IBGE, 2010), a adoção de "wetland, aplicação no solo e plantas aquáticas" para tratamento de esgoto sanitário no país correspondia na época a apenas 7.2\% (109 unidades) de instalações de tratamento. Essa situação pode estar relacionada principalmente à limitada disseminação de tecnologia fora das instituições de ensino e pesquisa.

Atualmente, existem artigos de revisão bibliográfica sobre o uso de wetlands construídas para tratamento de águas residuárias no Brasil, destacando-se os levantamentos realizados por Sezerino et al. (2015) e Machado et al. (2017). No entanto, a maioria dos trabalhos sintetizados por eles são artigos publicados em periódicos e eventos científicos (simpósios, conferências e congressos), negligenciando estudos de pós-graduação (dissertações e teses). Além do mais, os levantamentos citados não contemplaram toda a produção acadêmica (artigos) brasileira, desprezando informações de diversos trabalhos.

A reduzida disseminação de dados e informações obtidas nas pesquisas de pós-graduação brasileira resulta na retenção e baixa disseminação de conhecimentos ligados à tecnologia das wetlands construídas, o que provoca a redução do seu uso fora do alcance das instituições de ensino e pesquisa. Muitas das pesquisas de mestrado ou doutorado não culminam em publicações em periódicos ou eventos científicos, o que limita ainda mais a divulgação do conhecimento gerado nesses estudos.

Neste contexto, o presente trabalho teve como objetivo coletar informações sobre as pesquisas de wetlands construídas para tratamento de águas residuárias na pós-graduação (dissertações de mestrado e teses de doutorado) no Brasil.

\section{METODOLOGIA}

O levantamento dos trabalhos (dissertações e teses) desenvolvidos nos cursos de pós-graduação brasileiro sobre o uso de wetlands construídas para o tratamento de águas residuárias foi realizado conforme as etapas sequenciais: 
1. Busca no site de teses e dissertações da Coordenação de Aperfeiçoamento de Pessoal de Nível Superior (CAPES) (www.portaldetesescapes.gov.br), inserindo como palavras-chave de pesquisa os termos "alagados construídos" e "wetlands construídas".

2. Pesquisa nos repositórios de busca de teses e dissertações de todas universidades públicas brasileiras, utilizando-se também as palavras-chave "wetlands construídas" e "alagados construídos".

3. Busca de novas teses e dissertações citadas nos trabalhos encontrados nas etapas anteriores. O número de trabalhos encontrados nos mecanismos de busca foi alto (acima de 500). No entanto, nem todos foram incluídos no tema "wetlands construídas para o tratamento de águas residuárias". Assim, foi feita a seleção manual das dissertações e teses inseridas neste tema. Outra pré-seleção foi a escolha das pesquisas que desenvolveram rotinas experimentais (monitoramento dos processos de tratamento).

Ao todo, foram obtidos 274 trabalhos, sendo $74 \%$ de dissertações, correspondentes à 204 pesquisas, e $26 \%$ de teses, relativos à 70 pesquisas. No total, 21 não estavam disponíveis para download ou acesso às informações detalhadas da pesquisa. Assim, como estágio posterior deste estudo, os dados foram sintetizados a partir de 253 documentos.

De cada trabalho, foram coletados os seguintes dados: "instituição da pesquisa", "produção anual" (baseada na data da defesa da dissertação ou tese), "espécies vegetais utilizadas", "tipologia da água tratada", "período de monitoramento", "vazão de tratamento", "área superficial de tratamento", "unidades controle e plantada" "meio suporte utilizado", "nomenclatura adotada", "direção do fluxo hidráulico", "tipo de pré-tratamento" e "poluentes estudados".

Posteriormente, esses dados foram resumidos em forma gráfica e numérica para serem interpretados e discutidos. Os dados das "instituições de pesquisa", "produção anual", "espécies vegetais utilizadas", "tipologia da água tratada", "meio suporte utilizado", "nomenclatura adotada", "direção do fluxo hidráulico", "unidades controle e plantada", "tipo de prétratamento" e "poluentes estudados" foram analisados em termos numéricos e percentuais. Já para a análise do "período de monitoramento", "vazão de tratamento" e "área superficial de tratamento", foram adotadas faixas de valores.

\section{RESULTADOS E DISCUSSÃO}

\subsection{Instituição da pesquisa}

Foram produzidas teses e dissertações aplicando wetlands construídas no tratamento de águas residuárias em 43 diferentes universidades brasileiras. A região sudeste foi a que apresentou maior número (18 instituições; 42\%), seguida pelas regiões sul (13 instituições; 30\%), nordeste (6 instituições; $14 \%$ ), centro-oeste (5 instituições; $12 \%$ ) e norte (1 instituição; $2 \%$ ).

A Universidade de Campinas (UNICAMP) foi a que gerou maior quantidade de trabalhos (30), correspondendo a $11.9 \%$ do produzido no Brasil até então. Em segundo lugar, destaca-se a Universidade Federal de Viçosa (UFV) com 24 pesquisas (9.5\%). A Universidade Federal de Santa Catarina (UFSC) e a Universidade Estadual Paulista (UNESP) apresentaram cada uma 19 trabalhos (7.5\%).

Em termos de estado, São Paulo foi o que apresentou o maior número de produções (71), seguido por Minas Gerais (45), Santa Catarina (25) e Rio Grande do Sul (22).

Padrão similar a esse estudo foi observado em Machado et al. (2017), em que apenas 13\% dos trabalhos avaliados foram desenvolvidos fora das regiões sul-sudeste, o que demonstra que as 
pesquisas brasileiras sobre wetlands construídas aplicadas ao tratamento de águas residuárias são predominantemente oriundas dessas regiões.

A elevada desigualdade na quantidade de pesquisas sobre o tema entre as regiões geográficas brasileiras (as seis primeiras instituições no número de trabalhos são das regiões sul-sudeste, representando $46.5 \%$ das dissertações e teses produzidas no Brasil) pode estar relacionada a fatores como diferenças nas fontes de financiamento da pesquisa, qualidade dos cursos de pósgraduação, existência de líderes de grupos de pesquisa interessados no assunto, entre outros.

\subsection{Produção anual}

A primeira pesquisa brasileira em nível de pós-graduação aplicando wetlands construídas para tratamento de efluentes foi em 1989 (MANFRINATO, 1989). Segundo a Figura 1, somente em 1998 é que surgiram novas dissertações ou teses no Brasil com esse tema. Desde 1998 (até 2019), sempre houve pelo menos uma dissertação ou tese por ano no país sobre esse assunto.

Figura 1: Evolução do número de pesquisas ao longo dos anos sobre o tema abordado.

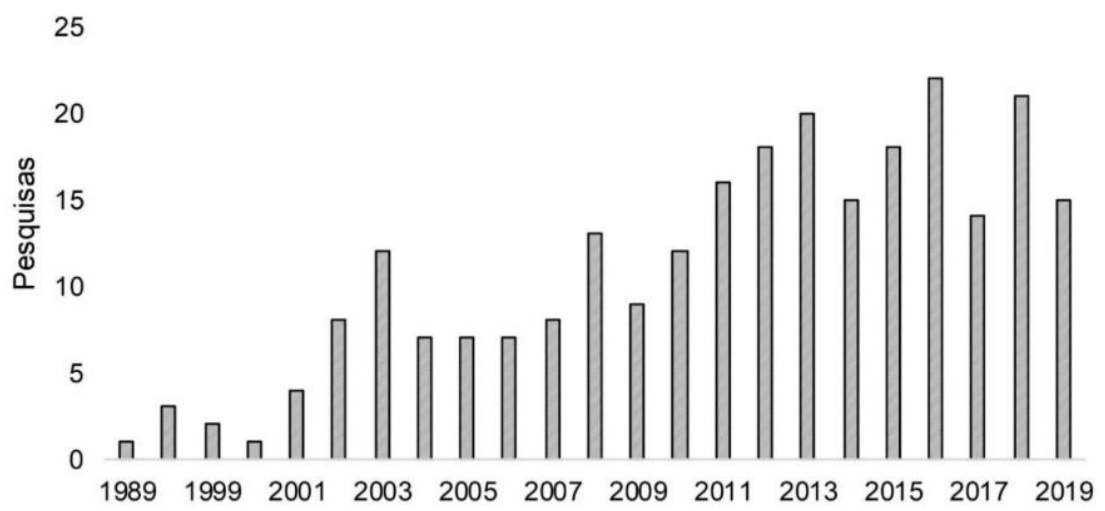

Ano do trabalho

Até o final da década de 1990 houveram poucos estudos sobre o tema no país. Na Europa e América do Norte, diversos trabalhos foram desenvolvidos principalmente após os anos 1980 (VYMAZAL, 2011), indicando que os estudos brasileiros sobre a temática são bastantes recentes comparados aos países desenvolvidos.

A partir de meados dos anos 2000 houve crescimento considerável na produção de dissertações e teses no país. Entre os anos de 2009 e 2013, o aumento ocorreu em taxa linear, sendo o ano de 2016 o que apresentou o maior número de obras (22). Entre 2008 e 2019, a produção acadêmica média foi de aproximadamente 16 dissertações ou teses por ano, enquanto a média entre 2001 e 2007 foi pouco mais de sete trabalhos por ano. O crescimento vertiginoso no número de pesquisas desenvolvidas no Brasil a partir dos anos 2000 pode ser atribuído a fatores como a melhoria do acesso a outras pesquisas (nacionais e internacionais) via internet, maior interesse de pesquisadores e grupos de pesquisa pela tecnologia, aumento da quantidade de eventos científicos, em nível nacional e internacional, para difusão do conhecimento sobre o tema, entre outros fatores.

O ano de 2019 pode vir a apresentar mais trabalhos, considerando-se que dissertações e teses produzidas neste ano podem ainda não terem sido publicadas pela CAPES ou pelos repositórios de pesquisa das universidades até o momento da busca realizada nesta pesquisa. 


\subsection{Espécies vegetais utilizadas}

O gênero vegetal Typha spp foi o principal utilizado nas dissertações e teses levantadas (Figura 2). Espécies desse gênero foram utilizadas em 92 trabalhos (36.4\% do total). Em segundo lugar, com presença em 35 dissertações e teses, destacaram-se as espécies do gênero Cyperus spp (13.8\%). Em terceiro lugar, a planta aquática flutuante Eichhornia crassipes (também conhecida como águapé) foi utilizada em 27 dissertações ou teses (10.7\% do total), predominantemente em sistemas de tratamento de escoamento superficial.

Figura 2: Principais espécies vegetais utilizadas nas pesquisas.

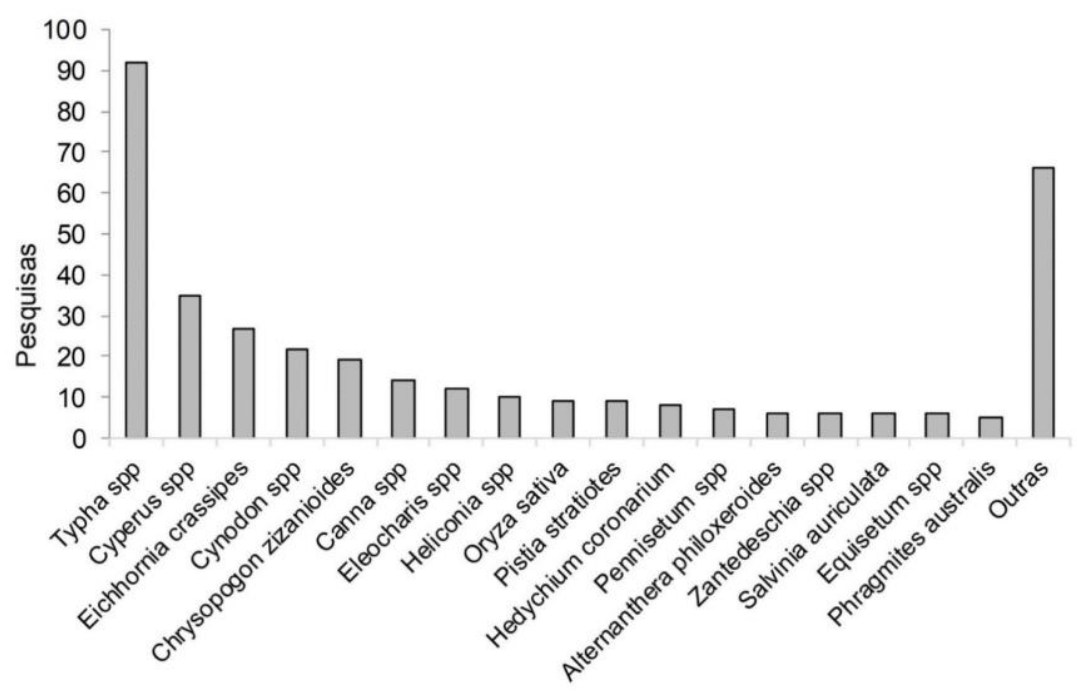

Condizente com esse trabalho, Sezerino et al. (2015) verificaram que o gênero Typha spp foi o mais utilizado, em 60\% dos estudos avaliados. Machado et al. (2017) e Zinato e Guimarães (2017) também avaliaram que esse gênero constituiu o mais adotado nos trabalhos avaliados, com $36 \%$ e $21 \%$ dos artigos estudados, respectivamente.

Os principais motivos da escolha do gênero Typha spp são: facilidade de obtenção (por apresentarem alta distribuição geográfica em climas tropicais), simplicidade no plantio e manutenção, rápido crescimento e adaptação ao ambiente de tratamento (incluindo resistência à presença de compostos tóxicos para outras plantas), além de alta produção de biomassa e remoção de poluentes (MAZZOLA et al., 2005; FIA et al., 2011; FIA et al., 2017).

Espécies do gênero Cyperus spp. são bem indicadas pela sua adaptabilidade a ambientes aquáticos e principalmente pela sua aparência estética (TRAVAINI-LIMA; SIPAÚBA-TAVARES, 2012; SEZERINO et al., 2012). No estudo de Sezerino et al. (2015), o gênero Cyperus spp apresentou apenas $7 \%$ dos trabalhos avaliados, correspondendo apenas à quarta posição, atrás dos gêneros Typha spp. (60\%), Eleocharis spp (14\%) e Zizaniopsis spp. (12\%). Já Zinato e Guimarães (2017) contabilizaram apenas $8 \%$ de espécies desse gênero em sua pesquisa.

A espécie flutuante Eichhornia crassipes é escolhida principalmente porque apresenta rápido crescimento, alta produção de biomassa e alta capacidade na remoção de poluentes (HENRYSILVA; CAMARGO, 2008; TRAVAINI-LIMA; SIPAÚBA-TAVARES, 2012).

Ao todo, 63 espécies ou gêneros diferentes foram encontrados nos trabalhos pesquisados. Essas incluem espécies que podem ser utilizadas na alimentação animal (Alternathera philoxeroides, 
Brachiaria spp, Cynodon spp, Urochloa mutica e outras), consumo humano (Zea mays - milho, Oryza sativa - arroz e Colocassia esculenta - inhame), fabricação de produtos de beleza (Chrysopogon zizanioides), paisagísmo (Cyperus spp, Hedychium coronarium, Zingiber spectabile, Hemerocallis flava e outros), uso medicinal (Polygonum punctatum, Mentha aquática e outros), aquarismo (Elodea densa, Cabomba caroliniana, Vallisnenia spirallis) e outros fins.

\subsection{Tipologia da água tratada}

Foram contabilizadas 36 diferentes tipologias de água tratada nas dissertações e teses levantadas (Figura 3). "Esgoto universitário" (proveniente de sanitários, lavatórios, pias de laboratórios, limpeza de pisos e outras fontes geradoras dentro das universidades) foi a tipologia mais utilizada nos estudos pesquisados, correspondendo a cerca de $20.6 \%$ (52) do total. Em segundo lugar, "esgoto sanitário urbano" (oriundo de cidades e com possível presença de água de chuva) esteve presente em 20.2\% (51) dos trabalhos pesquisados. "Esgoto descentralizado" (água residuária com características domésticas e vazão inferior a 5,000 L/d) foi utilizado em 9.1\% (23) dos estudos, ocupando o terceiro lugar no ranking. "Efluente de suinocultura" e "lixiviado de aterro sanitário" foram o quarto e quinto tipo de água residuária mais utilizada nas pesquisas, respectivamente.

Figura 3: Principais tipologias de água tratada das pesquisas.

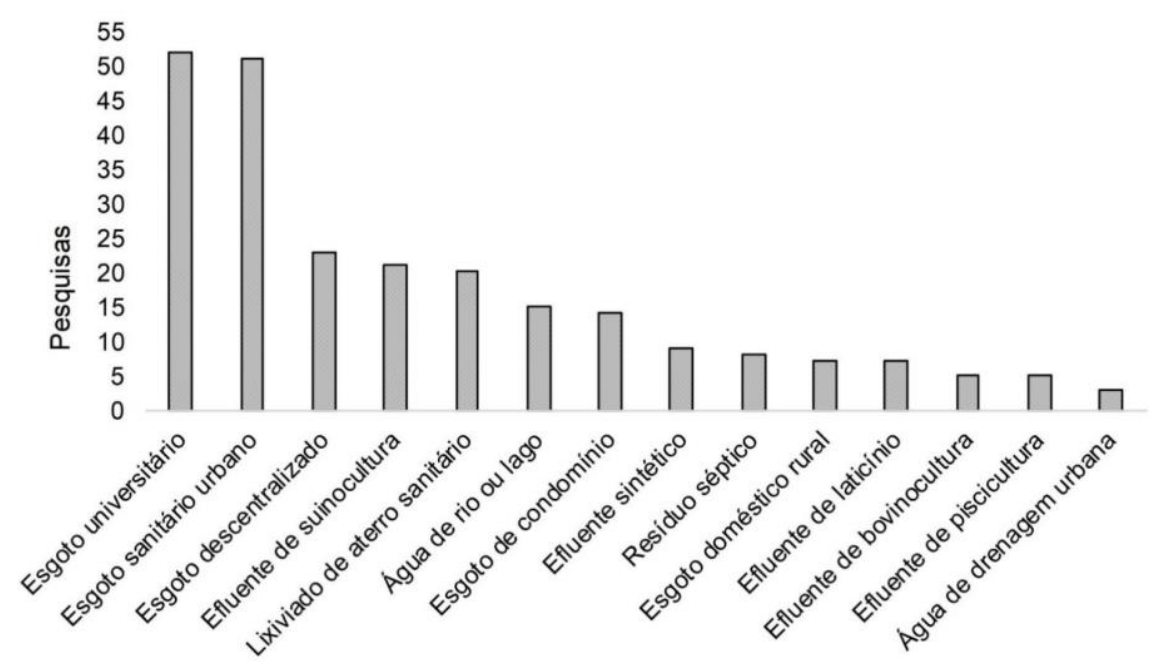

Outras matrizes utilizadas nas pesquisas foram: água de rios e lagos, esgoto de condomínio (gerado em residenciais com vazão de 5,000 L/d ou superior, rede coletora própria e tratamento descentralizado), efluente sintético (produzido em laboratório), resíduos sépticos, esgoto doméstico rural (gerados em moradias localizadas na área rural), entre outros.

Os seguintes tipos de águas residuárias podem ser citados como incomuns: efluentes de atividades aquícolas (incluindo piscicultura e maricultura), de ranicultura (criação de rãs), de fecularia, de curtume e de criação de camarões.

Em primeiro lugar nessa pesquisa, o esgoto universitário (20.6\%) avaliado possui característica doméstica na maior parte do tempo (baixa carga orgânica e ausência de poluentes tóxicos metais pesados, por exemplo). No entanto, a contribuição dos resíduos líquidos de laboratório pode resultar em conteúdo orgânico elevado, nutrientes e compostos potencialmente tóxicos 
no esgoto a ser tratado e descartado no ambiente. Os levantamentos encontrados na literatura (SEZERINO et al, 2015; MACHADO et al, 2017; ZINATO; GUIMARÃES, 2017) não fizeram diferenciação entre esgoto universitário e outras fontes de esgoto doméstico.

A maioria das pesquisas utilizando esgoto sanitário urbano (20.2\%) foi resultado de parcerias entre instituições de ensino e pesquisa com concessionárias locais de saneamento, que forneceram esgoto para o estudo. Segundo Zinato e Guimarães (2017), 19\% dos trabalhos avaliados utilizaram esgoto sanitário urbano, resultado bastante similar ao encontrado nessa pesquisa. Machado et al. (2017) encontraram percentual maior, 27.3\%. Já Sezerino et al. (2015) estimou $62 \%$ de estudos com esgoto sanitário ou doméstico.

\subsection{Período de monitoramento}

Dentre as pesquisas encontradas, quanto ao período de acompanhamento das wetlands construídas, aproximadamente $65 \%$ (164) das dissertações e teses analisadas foram monitoradas (análise laboratorial dos parâmetros de qualidade de efluentes) entre três e doze meses. Cerca de $19 \%$ (47) dos estudos tiveram tempo de monitoramento do sistema de tratamento superior a 12 meses. O tempo de acompanhamento inferior a três meses correspondeu a aproximadamente $16 \%$ (42) dos trabalhos analisados.

O tempo de monitoramento do sistema de tratamento superior a 12 meses deve-se principalmente à pesquisa em nível de doutorado, apresentando assim período maior de coleta de dados. O período inferior a 12 meses corresponde geralmente ao tempo gasto na condução das atividades experimentais do nível de mestrado de pós-graduação no Brasil.

Maior período de acompanhamento das variações da qualidade da água residuária no sistema de tratamento é elemento essencial para obter dados mais representativos sobre a realidade estudada e para tirar conclusões sobre o processo. Quanto maior o período de monitoramento, mais confiáveis os dados e melhor a compreensão (e representatividade) do fenômeno estudado. Também, é necessário monitorar o sistema de tratamento até que esse apresente certo equilíbrio ou estabilização, de forma que estudos com períodos de monitoramento muito curtos podem não apresentar resultados representativos com a realidade de longo prazo.

\subsection{Vazão de tratamento}

Verificou-se que as vazões de águas residuárias inferiores a 1,000 L/d corresponderam a 50\% (127) dos estudos analisados. O segundo fluxo mais utilizado foi na faixa 1,000 - 5,000 L/d, 18\% (46) do total. A faixa de vazão de 10,000 a 50,000 L/d esteve presente em $17 \%$ (42) das dissertações e teses analisadas, enquanto fluxos superiores a $50,000 \mathrm{~L} / \mathrm{d}$ corresponderam a $9 \%$ (24) e as vazões entre os valores de 5,000 a $10,000 \mathrm{~L} / \mathrm{d}$ representaram $6 \%$ (14).

$\mathrm{O}$ uso de vazões de esgoto inferiores a $1,000 \mathrm{~L} / \mathrm{d}$ (principal utilizado) são explicadas pelo fato de muitas delas terem sido desenvolvidas em escala piloto (não utilizando todo esgoto disponível) ou serem responsáveis pelo tratamento de águas residuárias de poucas pessoas (esgoto descentralizado ou rural), o que implica em leitos de tratamento menores.

Embora haja muitos estudos em escala piloto e em escala laboratorial, enfatiza-se que o maior uso de baixos volumes aplicados diariamente está correlacionado ao fato de que os ambientes alagados construídos são mais difundidos para atender poucas residências (consequentemente, baixas vazões), tratando águas residuárias geradas no contexto descentralizado (CRITES; TCHOBANOGLOUS, 1998; KADLEC; WALLACE, 2009).

A faixa de vazão de 10,000 a $50,000 \mathrm{~L} / \mathrm{d}$ esteve presente em $17 \%$ (42) das pesquisas, enquanto fluxos superiores a 50,000 L/d corresponderam a $9 \%(24)$ dos estudos avaliados. Esses dados 
indicam que as wetlands construídas também ocorreram em pesquisas em escala real, tratando grandes volumes de águas residuais. Destacam-se os trabalhos de Preussler (2014) e TRAVAINILIMA (2011) com fluxos médios de 720,000 L/d e 848,400 L/d, respectivamente.

\section{7 Área superficial de tratamento}

Cerca de $33 \%$ (83) dos trabalhos analisados apresentaram leitos de tratamento com áreas de superfície inferior a $5 \mathrm{~m}^{2}$. A faixa de valores de 5 a $50 \mathrm{~m}^{2}$ correspondeu a $40 \%$ (102) do total de pesquisas, enquanto os valores entre 50 e $100 \mathrm{~m}^{2}$ e acima de $100 \mathrm{~m}^{2}$ corresponderam a $10 \%$ (25) e $17 \%$ (43), respectivamente.

O estudo de Sezerino et al. (2015) indica que apenas $20 \%$ dos trabalhos analisados utilizaram área superficial de tratamento inferior a $5 \mathrm{~m}^{2}$, resultado inferior ao realizado nesse levantamento (27\%). No entanto, sua pesquisa analisou apenas as wetlands construídas horizontais de fluxo subsuperficial. Já Zinato e Guimarães (2017) obtiveram valor similar ao encontrado nessa pesquisa, 30\%, para leitos com área superficial de até $5 \mathrm{~m}^{2}$.

Sezerino et al. (2015), Machado et al. (2017) e Zinato e Guimarães (2017) estimaram respectivamente, $45,61.4$ e $61 \%$, de áreas superficiais de leitos com valores acima de $10 \mathrm{~m}^{2}$, resultados similares a essa pesquisa, $59 \%$.

Esses resultados, juntamente com os dados de vazão tratada, confirmam que as wetlands construídas podem ser aplicadas no tratamento de águas residuárias de pequenos e grandes geradores. Destaca-se o estudo de Bidone (2007), com área superficial de leito de tratamento de $5,000 \mathrm{~m}^{2}$.

\subsection{Unidades controle e plantada}

Leitos com vegetação corresponderam a $78 \%$ (197) do total de unidades de tratamento implantadas nas pesquisas, enquanto as células sem vegetação (denominadas como controle) a $22 \%$ (56) dos leitos, indicando relação superior a três entre unidades com vegetação e controle. Qualquer que seja a nomenclatura adotada, mais de $90 \%$ dos estudos utilizaram pelo menos uma célula de tratamento controle para avaliar a influência da vegetação no tratamento estudado, o que demonstra sua importância no desenho experimental da pesquisa.

As células controle, embora sem vegetação, são enquadradas por alguns autores como alagados construídos (FIA et al., 2017; MATOS et al., 2017). Entretanto, nesses ambientes não há interação meio suporte-planta-microrganismo, o que confere sua desqualificação quanto ao uso da terminologia de wetlands construídas. De todo modo, sua importância é considerável nas pesquisas, pois funciona como elemento comparativo da efetividade da vegetação no sistema de tratamento.

\subsection{Meio suporte utilizado}

Predominantemente, a brita (em tamanhos variados) foi o principal material usado como suporte nas wetlands construídas para o tratamento de águas residuárias (Figura 4).

Somente a brita foi adotada por $41 \%(104)$ das dissertações e teses levantadas. A associação entre brita e areia foi implantada em $18 \%$ (45) dos estudos. Apenas $4 \%$ (11) das pesquisas utilizaram somente areia. Combinações de brita, areia e pedregulho corresponderam a $3 \%(8)$, a escória de mineração, o solo com brita, e outros representaram juntos 34\% (85). 
Ao todo, 50 combinações diferentes de meio suporte foram observadas. Além da brita e areia, pode-se citar a presença de meios como conchas de moluscos, bambu, palha de café, carvão, turfa, isopor, pneu picado, casca de arroz, garrafas PET e outros.

A pesquisa realizada por Sezerino et al. (2015) estimou a presença de brita na composição do material de suporte em 64\% das pesquisas avaliadas. Já Zinato e Guimarães (2017) avaliaram que em $52 \%$ dos estudos houve a presença desse material com meio suporte, o que atesta o resultado obtido no presente estudo: a brita é o principal meio suporte utilizado.

A principal vantagem do uso de brita como suporte é sua maior porosidade (consequentemente condutividade hidráulica maior), em relação a outros meios de suporte como a areia e argila (apresentam menor condutividade hidráulica). Além disso, o uso da brita reduz a possibilidade de colmatação do leito de tratamento, estimula a difusão de oxigênio dissolvido da atmosfera para o ambiente de tratamento, e promove a remoção de nitrogênio e matéria orgânica das águas residuárias. Por outro lado, tanto a brita quanto a areia tem pequena capacidade de troca de cátions (CTC), o que reduz a capacidade do sistema de tratamento de remover fósforo (KADLEC; WALLACE, 2009).

Figura 4: Substratos utilizados como meio suporte dos leitos de tratamento nas pesquisas.

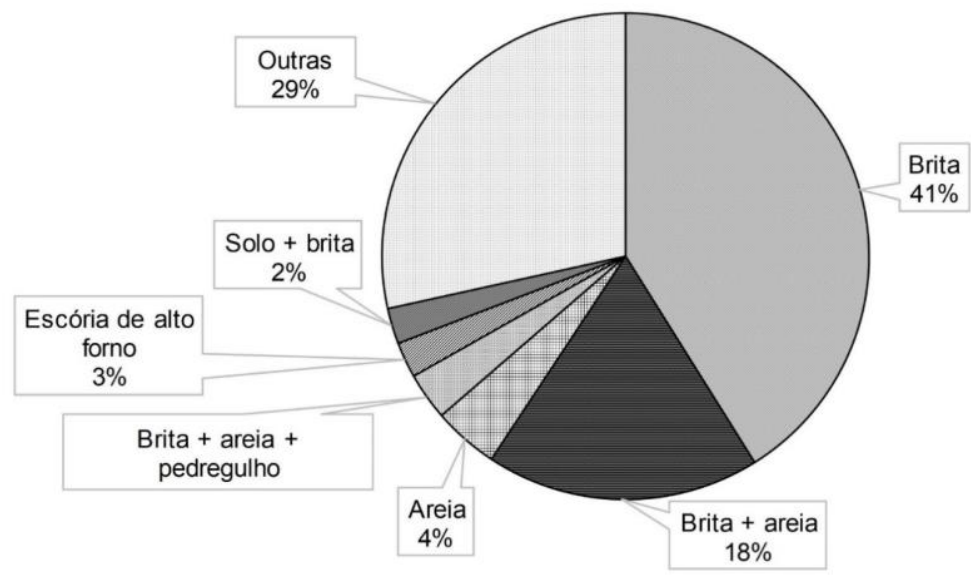

Substratos como argila e escória de mineração possuem alta CTC (alta capacidade de remoção de fósforo em efluentes), mas, por outro lado, possuem baixa porosidade e alto risco de entupimento de leito (MATOS et al., 2017), por isso, são pouco utilizadas.

\subsection{Direção do fluxo hidráulico}

Em geral, o tipo de fluxo hidráulico (vertical ou horizontal, superficial ou subsuperficial) mais adotado foi o subsuperficial vertical, com presença em $54 \%$ (138) dos estudos. Com relação somente à direção do escoamento, a maioria das dissertações e teses $(47 \% ; 119)$ analisadas adotou apenas unidades de tratamento com fluxo horizontal. Estudos utilizando apenas células de tratamento de fluxo vertical corresponderam a $34 \%$ (86) dos casos. No entanto, os sistemas com a presença de ambos os fluxos (híbridos) representaram 19\% (48) das pesquisas.

O uso exclusivo de células de tratamento com fluxo superficial correspondeu a $11.1 \%$ (28) dos estudos, enquanto que os trabalhos com unidades de fluxo apenas subsuperficial totalizaram $80.6 \%$ (204). Os sistemas híbridos (unidades de fluxo superficial e subsuperficial) representaram $8.3 \%(21)$ das dissertações e teses. 
Os sistemas de fluxo horizontal subsuperficial foram utilizados em $38 \%$ (95) das dissertações e teses levantadas. As unidades de escoamento horizontal superficial foram adotadas em apenas $8 \%$ (20) do total de pesquisas.

Como o Brasil é um país tropical, com alta incidência de doenças transmitidas por vetores, especialmente aquelas causadas por mosquitos, recomenda-se a adoção de sistemas de tratamento de fluxo subsuperficial (sem contato direto do meio líquido com a atmosfera) (SAEED; SUN, 2012). Sezerino et al. (2015) avaliaram somente wetlands construídas de fluxo horizontal subsuperficial, enquanto Zinato e Guimarães (2017) calcularam que $69 \%$ dos sistemas analisados apresentaram tal fluxo de escoamento.

Os sistemas de fluxo vertical são adequados e utilizados principalmente para estimular processos aeróbicos (como nitrificação e degradação aeróbia de matéria orgânica) no meio líquido através da introdução de oxigênio dissolvido (KADLEC; WALLACE, 2009). Esses sistemas possuem características predominantemente anaeróbias (desde que não seja introduzido artificialmente oxigênio dissolvido no meio), o que pode desenvolver processos de desnitrificação, metanogênese e sulfetogênese (SAEED; SUN, 2012; VYMAZAL, 2013). Cerca de $33.4 \%$ dos trabalhos analisados por Machado et al. (2017) foram com células de fluxo vertical. Por ter contato direto com a atmosfera, os sistemas superficiais não são os mais adequados e utilizados, pois podem ser responsáveis por atrair vetores de doenças (principalmente mosquitos) e gerar odores desagradáveis. Zinato e Guimarães (2017) estimaram que apenas $9 \%$ dos estudos adotaram tal tipo de fluxo.

\subsection{Nomenclatura adotada}

Embora a terminologia "wetlands construídas" seja conhecida internacionalmente, outros nomes foram adotados nos títulos de dissertações e teses do presente levantamento. Ao todo, foram encontradas 14 denominações diferentes nos trabalhos consultados (Figura 5).

Figura 5: Terminologias adotadas para os sistemas de tratamento nas dissertações e teses avaliadas.

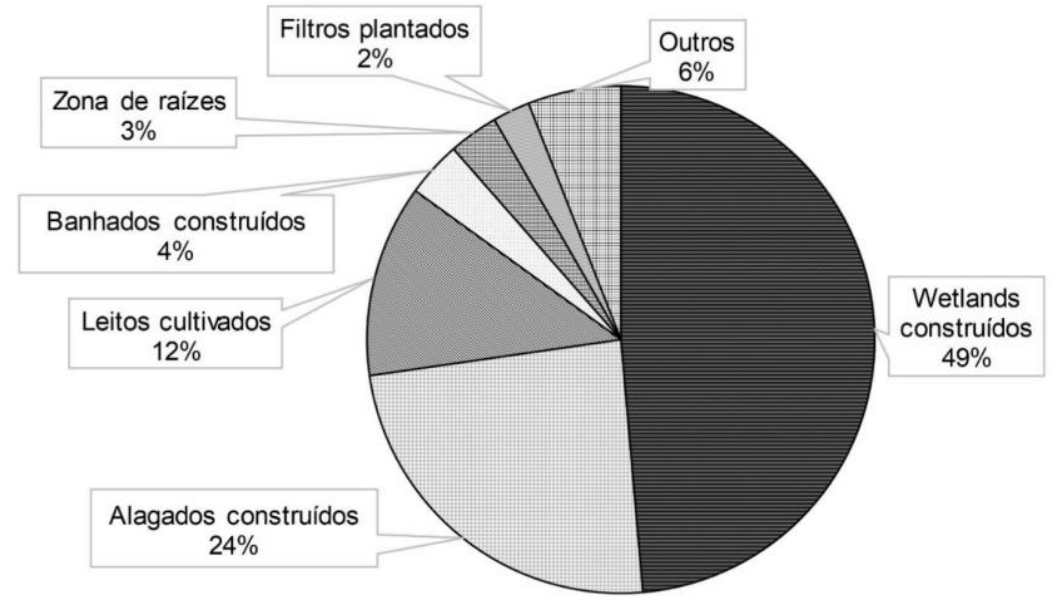

"Wetlands construídas (os)" foram utilizadas no título de $49 \%$ (123) das pesquisas, sendo desse valor $52.0 \%$ para "wetlands construídas" e $48.0 \%$ para "wetlands construídos". Em segundo lugar, em $24 \%$ (61) das pesquisas, foi adotado o termo "alagados construídos" para se referir ao tema deste artigo. 
O terceiro nome mais comum adotado pelos autores foi "leitos cultivados", usado em $12 \%$ dos trabalhos (31). "Banhados construídos" e "zona de raízes" estavam presentes, cada uma, em 4\% (9) e $3 \%$ (8) dos estudos, respectivamente. Já "filtros plantados" corresponderam a $2 \%$ (6) das pesquisas. Além desses, também foram encontradas terminologias como "terras úmidas", "leito de macrófitas", "sistema edafofitopedológico", "áreas alagadiças", "zona alagadiça", "biofiltro com macrófitas", "zonas úmidas construídas" e "constructed wetlands", todas juntas somando $6 \%$ do total (15).

A terminologia "wetlands construídas", principal adotada pelos autores brasileiros, é fortemente influenciada pela origem do termo em inglês. O nome "alagado" consiste em uma das traduções (do inglês para português) do termo "wetland", implicando que ambas terminologias são as mesmas, mas apenas em diferentes idiomas.

Percebe-se que a nomenclatura adotada em cada trabalho tem grande influência da cultura na instituição de desenvolvimento da pesquisa. Mais de $80 \%$ dos trabalhos desenvolvidos na Universidade Federal de Viçosa adotaram o termo "alagados construídos", enquanto mais de $70 \%$ das teses e dissertações produzidas na Universidade Estadual de Campinas usaram o nome "leitos cultivados". Essa nomenclatura "leitos cultivados' fornece a ideia de cultivar a vegetação em um ambiente de tratamento.

O regionalismo também pode ser observado na escolha do nome para o sistema de tratamento. O termo "banhados construídos" foi utilizado quase que exclusivamente por instituições sobre a influência da cultura gaúcha, como a Universidade Federal do Rio Grande do Sul e a Universidade Federal do Mato Grosso do Sul. O termo "banhados" para os gaúchos também tem o significado de wetlands.

\subsection{Tipo de pré-tratamento}

A Figura 6 apresenta a relação entre a quantidade de dissertações e teses e alguns dos tratamentos adotados previamente às wetlands construídas.

Figura 6: Unidades de tratamento adotado previamente às wetlands construídas nas pesquisas.

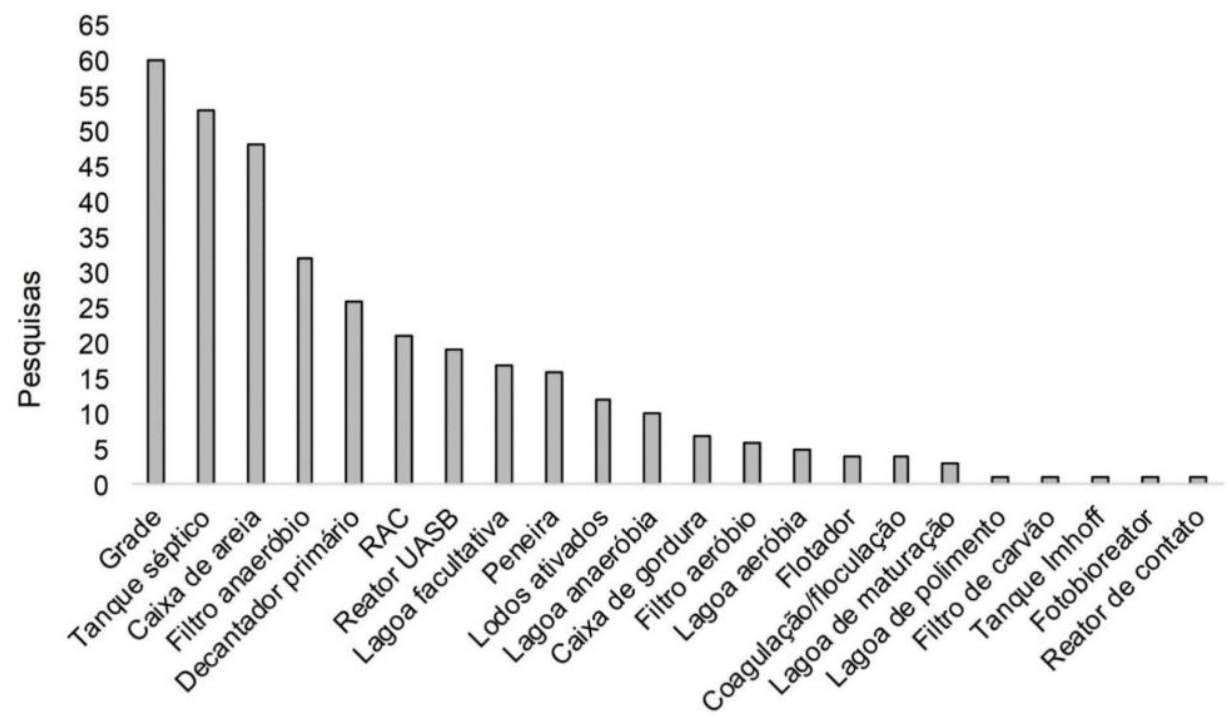


Os sistemas de wetlands construídas utilizados nos trabalhos analisados foram aplicados principalmente em nível de tratamento terciário (50.2\% dos casos - 126 trabalhos), após unidades de tratamento secundário (predominantemente biológicas).

Em nível terciário, as wetlands construídas foram posteriormente implantadas a Reator Anaeróbio de Fluxo Ascendente (UASB), reator anaeróbio compartimentado (RAC), filtro anaeróbio e lagoa anaeróbia, e sistemas aeróbios (lodos ativados, filtro aeróbio, fotobiorreatores, contator biológico rotativo, lagoas aeradas e facultativas). Reatores UASB e RAC, filtros anaeróbios e lagoas anaeróbias foram implantados (isoladamente ou em conjunto com outras unidades de pré-tratamento) em 7.5\%, 8.3\%, 12.6\% e $4.0 \%$ dos estudos avaliados, respectivamente. $\mathrm{O}$ uso de unidades aeróbias como lodos ativados, filtro aeróbio, lagoas aeradas e lagoas facultativas foram utilizadas em $4.7 \%, 2.4 \%, 2.0 \%$ e $6.7 \%$ dos estudos, respectivamente.

Aproximadamente $20.3 \%$ (51) das dissertações e teses analisadas utilizaram wetlands como unidades de tratamento no nível secundário, com a remoção de certa porcentagem de sólidos grosseiros, sedimentáveis e material flotável nas unidades anteriores. As wetlands construídas foram implantadas após unidades como decantador primário, flotador, sistema de coagulação/floculação em $10.3 \%, 1.6 \%$ e $1.6 \%$ das pesquisas, respectivamente.

Apenas $6.8 \%$ (17) das pesquisas implementaram alagados construídos como unidades de tratamento no nível primário, com tratamento apenas preliminar (grade e/ou caixa de areia) anteriormente.

Parte das dissertações e teses analisadas, $22.7 \%$ do total (57 trabalhos), não adotaram nenhum tipo de pré-tratamento, aplicando águas residuárias in natura diretamente nos sistemas de wetlands construídas.

Algumas tipologias de água (lixiviado de aterro e águas superficiais dos rios, por exemplo) não possuem como características a presença de grande quantidade de sólidos sedimentáveis e em suspensão, reduzindo assim a necessidade de tratamento primário e preliminar $(22.7 \%$ dos estudos avaliados nessa pesquisa não utilizaram pré-tratamento). No estudo de Machado et al. (2017), 45.4\% das wetlands construídas foram adotadas como unidades de tratamento primário e apenas $22.7 \%$ como secundário. No presente levantamento, $50.2 \%$ das dissertações e teses levantadas indicaram wetlands construídas em nível de tratamento terciário, e 20.3\% como nível secundário. A divergência nos resultados desses autores com a presente pesquisa pode estar na adoção dos critérios para enquadramento das unidades de tratamento. Por exemplo, alagados construídos utilizados após tratamento em reator UASB podem ser considerados como nível secundário ou terciário, dependendo do enquadramento conceitual adotado pelo autor.

Destaca-se o uso de tanque séptico (presente em 20.9\% dos estudos aqui levantados) em sistemas de pequeno e médio porte (vazões inferiores a 5,000 L/d), realizando sedimentação e digestão parcial do poluente antes do tratamento pelas wetlands construídas. Zinato e Guimarães (2017) contabilizaram apenas $10 \%$ das pesquisas com essa unidade de prétratamento.

\subsection{Poluentes estudados}

Ao todo, 49 tipos de poluentes foram utilizados nos trabalhos avaliados. "Demanda química de oxigênio" (DQO) e "demanda bioquímica de oxigênio" (DBO) foram monitoradas em 172 (68\%) e 128 (51\%) estudos, respectivamente. "Carbono orgânico total" (COT) foi avaliado em apenas 18 estudos (7\%).

Níveis de "nitrogênio amoniacal" e "fósforo total" foram quantificados em 107 (42\%) e 116 (46\%) estudos, respectivamente, correspondendo aos principais nutrientes de qualidade da 
água analisados. "Nitrogênio total", "nitrogênio total Kjeldahl" (TKN), "nitrogênio orgânico", "nitrito" e "nitrato" foram monitorados em 70 (28\%), 51 (20\%), 2 (1\%), 75 (30\%) e 96 (38\%) trabalhos, respectivamente. "Fosfato total" e "ortofosfato" foram quantificados em 27 (11\%) e $23(9 \%)$ estudos, respectivamente.

Pelo menos um parâmetro da série de sólidos (total, sedimentável, dissolvido, suspenso, fixo ou volátil) foi utilizado em 111 (44\%) dissertações e teses. "Sólidos suspensos" e "sólidos sedimentáveis" foram os principais tipos de sólidos avaliados, correspondendo a cerca de $48 \%$ e $19 \%$ dos estudos com a presença de sólidos monitorados.

Do ponto de vista microbiológico, a presença e remoção de bactérias pelos sistemas de tratamento foi avaliada em 63 (25\%) trabalhos. "Coliformes totais", "coliformes termotolerantes" e "Escherichia coli" foram os principais monitorados, funcionando como indicadores da presença de organismos patogênicos na água. Também, a presença de "Enterococcus faecalis", "Enterovirus", "Giardia", "Ascaris" e "Cryptosporidium" também foi avaliada em alguns estudos. Outros trabalhos também avaliaram a presença de fungos, algas e protozoários no ambiente de zonas úmidas.

"Condutividade elétrica", "temperatura" de água ou esgoto, "turbidez", "alcalinidade" e "oxigênio dissolvido" foram medidas em 83 (33\%), 72 (28\%), 69 (27\%), 57 (23\%) e 65 (26\%)

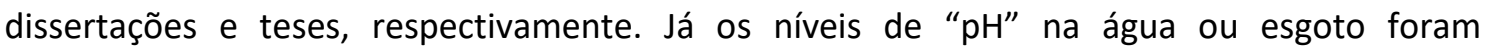
contabilizados em 149 (59\%) estudos.

Outros parâmetros importantes de qualidade da água foram monitorados, mas em número menor de estudos: "metais pesados", "pesticidas", "hormônios", "antibióticos", "antiinflamatórios", "fenóis", "hidrocarbonetos" e outros.

Em avaliações convencionais da eficiência de sistemas de tratamento de águas residuárias, o estudo do teor de matéria orgânica e nutrientes no esgoto são essenciais, por serem estimadores básicos dos níveis de impactos ambientais em corpos hídricos, por exemplo. Devido à facilidade analítica e reduzidos custos, o monitoramento da remoção de matéria orgânica pelas áreas alagadas construídas foi realizado predominantemente pelos parâmetros DBO e/ou DQO. Já a avaliação de COT nas águas residuárias é demasiadamente onerosa e por isso, apresentou menor quantidade de estudos.

Compostos químicos pertencentes às séries de nitrogênio, fósforo e sólidos também foram avaliados em diversos trabalhos (embora em menor quantidade de estudos do que os indicadores de matéria orgânica) e são importantes estimadores da eficiência de tratamento e dos possíveis impactos ambientais decorrentes da aplicação de águas residuárias no meio ambiente.

Devido aos altos custos e dificuldades analíticas, a quantificação de metais, microrganismos, pesticidas, fármacos, hidrocarbonetos, antibióticos, hormônios e outros, foi realizado em apenas alguns trabalhos, o que dificulta a avaliação da efetividade dos sistemas de wetlands construídas no tratamento de águas residuárias contaminadas com esses poluentes.

\section{CONCLUSÃO}

O número de teses e dissertações desenvolvidas sobre aplicações de wetlands construídas no tratamento de águas residuárias no Brasil teve crescimento a partir do início dos anos 2000, com as instituições das regiões sul-sudeste respondendo pela maior parte do que foi produzido no país.

Espécies do gênero Typha spp e brita foram a vegetação e meio suporte mais utilizados nas pesquisas, respectivamente. Os tipos de águas poluídas mais utilizadas nos estudos foram 
esgotos universitário e urbano, sendo aplicados tanto em grande como em pequena escala (servindo de cidades a residências unifamiliares).

As nomenclaturas mais utilizadas para os sistemas estudados foram "wetlands construídas" e "alagados construídos", respectivamente.

Os sistemas de tratamento estudados operaram predominantemente nos níveis secundário e terciário, avaliando principalmente a remoção de matéria orgânica e nutrientes das águas residuárias.

\section{REFERÊNCIAS BIBLIOGRÁFICAS}

BIDONE, Ricardo Figueira. Tratamento de lixiviado de aterro sanitário por um sistema composto por dois filtros anaeróbios seguidos de banhados construídos: estudo de caso - Central de Resíduos do Recreio, em Minas do Leão, RS. Dissertação (Mestrado em Engenharia - Hidráulica e Saneamento) - Universidade de São Paulo, São Carlos, Brasil, 2007.

CRITES, Ron; TCHOBANOGLOUS, George. Small and decentralized wastewater management systems. WCB/McGraw-Hill, EUA, 1998.

FIA, Fátima Resende Luiz et al. Remoção de nutrientes por Typha latifolia e Cynodon spp. cultivadas em sistemas alagados construídos. Ambi-Agua 6(1), 77-89, 2011.

FIA, Fátima Resende Luiz et al. Efeito da vegetação em sistemas alagados construídos para tratar águas residuárias da suinocultura. Engenharia Ambiental e Sanitária 22(2), 303-311, 2017.

HENRY-SILVA, Gustavo Gonzaga; CAMARGO, Antonio Fernando Monteiro. Tratamento de efluentes de carcinicultura por macrófitas aquáticas flutuantes. Revista Brasileira de Zootecnia 37(2), 181-188, 2008.

IBGE - Instituto Brasileiro de Geografia e Estatística. Pesquisa Nacional de Saneamento Básico - 2008. Ministério do Planejamento, Orçamento e Gestão, Rio de Janeiro, RJ, Brasil, 2010.

KADLEC, Robert; WALLACE, Scott. Treatment wetlands. Boca Raton: CRC Press, EUA, 2009.

MACHADO, Ana Isabel et al. Overview of the state of the art of constructed wetlands for decentralized wastewater management in Brazil. Journal of Environmental Management 187, 560-570, 2017.

MANFRINATO, Eneida Salati. Avaliação do método edafo-fitodepuração para tratamento preliminar de águas. Dissertação (Mestrado em Agronomia) - Universidade de São Paulo, Piracicaba, Brasil, 1989.

MATOS, Mateus Pimentel et al. Difficulties and modifications in the use of available methods for hydraulic conductivity measurements in highly clogged horizontal subsurface flow constructed wetlands. Water Science and Technology 76(7-8), 1666-1675, 2017.

MAZZOLA, Marcelo; ROSTON, Denis; VALENTIM, Marcelus. Uso de leitos cultivados de fluxo vertical por batelada no pós-tratamento de efluente de reator anaeróbio compartimentado. Revista Brasileira de Engenharia Agrícola e Ambiental 9(2), 276-283, 2005.

PREUSSLER, Karla Heloise. Avaliação de um sistema de wetlands no tratamento de lixiviado de aterro. Tese (Doutorado em Engenharia Civil) - Universidade Federal do Rio de janeiro, Rio de Janeiro, Brasil, 2014.

SAEED, Tanveer; SUN, Guangzhi. 2012. A review on nitrogen and organics removal mechanisms in subsurface flow constructed wetlands: Dependency on environmental parameters, operating conditions and supporting media. Journal of Environmental Management 112, 429-448, 2012.

SALATI JÚNIOR, Eneas; SALATI, Eneas; SALATI, Eneida. Wetlands projects developed in Brazil. Water Science and Technology 40(3), 19-25, 1999. 
SEZERINO, Pablo Heleno. et al. Constructed wetlands and sand filter applied as onsite post-treatment of anaerobic effluent. Water Practice Technology 7, 1-9, 2012.

SEZERINO, Pablo Heleno. et al. Experiências brasileiras com wetlands construídos aplicados ao tratamento de águas residuárias: parâmetros de projeto para sistemas horizontais. Engenharia Sanitária e Ambiental 20(1),151158, 2015.

TRAVAINI-LIMA, Fernanda. Capacidade de retenção e dinâmica de um wetland construído no tratamento de águas residuais. Dissertação (Mestrado em Aquicultura) - Universidade Estadual Paulista, Jaboticabal, Brasil, 2011.

TRAVAINI-LIMA, Fernanda; SIPAÚBA-TAVARES, Lúcia Helena. Efficiency of a constructed wetland for wastewaters treatment. Acta Limnologica Brasiliensia, 24(3), 255-265, 2012.

VYMAZAL, Jan. Constructed wetlands for wastewater treatment: five decades of experience. Environmental Science and Technology 45, 61-69, 2011.

VYMAZAL, Jan. The use of hybrid constructed wetlands for wastewater treatment with special attention to nitrogen removal: A review of a recente development. Water Research 47(14), 4795-4811, 2013.

ZINATO, Thamires Maria Campos; GUIMARÃES, Márcia Maria. Estudo sobre a utilização de "wetlands" construídas para tratamento de águas residuárias no Brasil. VIII Congresso Brasileiro de Gestão Ambiental, novembro 27-30, Campo Grande, Brasil, 2017. 